\title{
Microangiopatie trombotiche: classificazione e diagnosi differenziale
}

\author{
Giulia Antognoli, Lino Cirami \\ SOD Nefrologia e Dialisi, AOU Careggi, Firenze
}

\begin{abstract}
Thrombotic microangiopathies: classification and differential diagnosis
Thrombotic microangiopathies (TMA) are a group of clinical conditions mainly characterized by low platelet count, hemolytic anemia and organ damage. TMA can be acquired or hereditary and classified according to their etiology. Both males and females can be affected although different prevalence in age is observed and related to the subgroup. Hyperactivation of the alternative pathway of complement cascade, independently from its etiology, is accountable for microvascular thrombosis and its related clinical manifestations. The onset of TMA is often aggressive and it can be difficult to make an early correct differential diagnosis. It is crucial to perform specific tests at clinical presentation to help the clinician in making the diagnosis and to begin a better and more specific treatment. Research of genetic mutations is helpful to identify genetic TMA, sporadic or familiar, but not for the diagnosis at clinical onset. Because of TMA rarity, patients should be promptly sent to the attention of hematologists. New approaches on diagnosis and therapies have improved the prognosis of TMA especially in adults but further studies are needed.
\end{abstract}

Keywords: ADAMTS13, Hemolytic uremic syndrome, Thrombotic microangiopathy, Thrombotic thrombocytopenic purpura

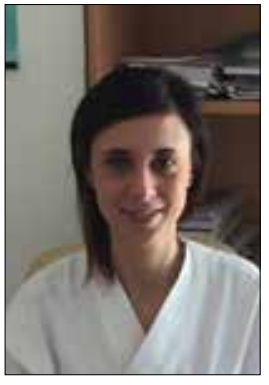

Giulia Antognoli

\section{Introduzione}

Le microangiopatie trombotiche (MAT) sono un gruppo di patologie caratterizzate dalla presenza di anemia emolitica microangiopatica, trombocitopenia e trombosi microvascolare a cui consegue un danno d'organo di entità variabile. Tali forme possono essere acquisite o ereditarie, a esordio acuto o progressivo e manifestarsi sia in età pediatrica che in età adulta. Esse presentano quadri clinici parzialmente sovrapponibili che, anche se attraverso diversi meccanismi patogenetici, determinano un danno a carico del microcircolo di vari organi e sistemi.

Accepted: April 6, 2016

Published online: August 3, 2016

Indirizzo per la corrispondenza:

Dr. Lino Cirami

SOD Nefrologia e Dialisi

AOU Careggi

Largo Brambilla 3

50134 Firenze

ciramil@aou-careggi.toscana.it

\section{Classificazione delle microangiopatie trombotiche}

Negli anni sono stati eseguiti numerosi tentativi di classificare le varie forme di MAT. Fra i più recenti ricordiamo quello dell'European Pediatric Research Group, pubblicato nel 2006 (Tab. I) (1), che tiene conto dell'eziologia, nota o sconosciuta, delle manifestazioni cliniche e dell'eventuale associazione con altre condizioni cliniche. Più recentemente JN George e CM Nester hanno, invece, proposto una nuova suddivisione in forme primitive e secondarie; le prime, a loro volta, sono state distinte in ereditarie e acquisite (Tabb. IIA e IIB) (2).

Le forme primitive riconoscono le MAT da deficit di ADAMTS13 (A Disintegrin And Metalloprotease with ThromboSpondin type 1 domain 13) e quelle complemento-mediate a genesi sia ereditaria che acquisita, mentre le forme metabolismo-mediate e coagulazione-mediate possono essere determinate esclusivamente geneticamente. Tra le MAT primitive sono comprese, inoltre, forme scatenate da tossinfezione da Shiga-toxin (Sindrome Emolitico Uremica Tipica o SEU D+ o ST-SEU) o dall'assunzione di farmaci.

Le forme secondarie sono, in genere, associate a infezioni sistemiche, a patologie neoplastiche, alla gravidanza (Preeclampsia, Eclampsia e Sindrome HELLP), a quadri di ipertensione maligna, a malattie autoimmuni (LES, Sclerosi Sistemica, Sindrome da Anticorpi Anti-Fosfolipidi) e al trapianto di organi solidi o di midollo. 
Nel 1924, Moschowitz descrisse, per la prima volta, una forma di MAT associata a sintomi neurologici e a interessamento cardiaco (3). Essa fu successivamente denominata Porpora Trombotica Trombocitopenica (PTT), che è stato dimostrato essere dovuta a un difetto, quantitativo o qualitativo, acquisito o ereditario, di una metallo-proteinasi secreta dalle cellule endoteliali, denominata ADAMTS13, con funzione di clivaggio dei multimeri circolanti del fattore di von Willebrand (vWF). La mancata formazione di monomeri o di dimeri di quest'ultimo

TABELLA I - Classificazione delle MAT in base all'eziologia

\begin{tabular}{ll}
\hline Microangiopatia trombotica provocata da \\
\hline - & Infezioni \\
- & Mutazioni genetiche o alterazioni acquisite della cascata del \\
- $\quad$ Deficit genetico o acquisito di ADAMTS13 \\
- $\quad$ Mutazioni del gene che codifica per la cobalamina \\
\hline
\end{tabular}

\section{Microangiopatia trombotica provocata da}

- HIV

- Neoplasie e relativi trattamenti

- Condizioni associate alla gravidanza (pre-eclampsia, eclampsia e HELLP syndrome) ed uso di contraccettivi orali

- $\quad$ Patologie autoimmuni (LES e APS)

- Glomerulopatie

- Causa sconosciuta (familiare o acquisita)

TABELLA IIA - MAT geneticamente determinate favorisce la genesi di microtrombi occludenti il lume vascolare determinata dalla "cattura" delle piastrine circolanti. La forma ereditaria di questa malattia (Sindrome di Upshaw-Schulman) è dovuta alla mutazione, in omozigosi o eterozigosi composta, del gene che codifica per ADAMTS13 (9q34), mentre la forma acquisita è associata alla presenza di anticorpi diretti contro questo enzima. La presenza di un'attività ridotta di ADAMTS13 (<10\%) o di anticorpi circolanti diretti contro ADAMTS13, associata a un prevalente interessamento neurologico, depone per una diagnosi di PTT; raro o minimo è il riscontro di danno d'organo renale in questi casi.

La SEU atipica (o MAT complemento-mediata) rappresenta il 5-10\% di tutte le SEU. Negli USA, l'incidenza stimata della patologia è di 2 casi per milione (4), includendo anche le forme secondarie a infezione da Streptococcus Pneumoniae. In Europa, invece, l'incidenza annuale, nella popolazione con età inferiore ai 18 anni, è stimata intorno a 3.3 casi per milione di abitanti, mentre tassi inferiori sono stimati per gli adulti (5). La malattia si può manifestare in qualsiasi periodo della vita, ma è prevalente in età pediatrica, dove è stata descritta un'incidenza uguale nei due sessi (6); in età adulta, è, invece, prevalente nel sesso femminile (7).

Si distinguono forme genetiche, acquisite o idiopatiche di MAT complemento-mediata. La variante genetica può essere familiare, quando la mutazione è presente in più componenti della stessa famiglia, oppure sporadica, se interessa un solo membro. Mutazioni tipo "loss of function" a carico dei geni che codificano per molecole regolatrici (CFH, MCP,

\section{Microangiopatie trombotiche geneticamente determinate}

Deficit di ADAMTS-13 (PTT)

Iperattivazione della via alterna del complemento

Attivazione della coagulazione

Attivazione della coagulazione (forme metabolismo-mediate)

\section{Causa}

Mutazione del gene che codifica per ADAMTS13 (9q34)

Mutazione dei geni $\mathrm{CFH}, \mathrm{CFI}, \mathrm{CFB}, \mathrm{C} 3$

Mutazioni di DGKE, PLG e THBD

Mutazione in omozigosi di MMACHC (MetilMalonic ACiduria and Homocystinuria type $\mathrm{C}$ protein)

TABELLA IIB - MAT acquisite

\section{Microangiopatie trombotiche geneticamente determinate}

Deficit di ADAMTS13 (PTT)

Tossinfezione da Shiga-toxin (ST-SEU)

Farmaci

\section{Causa}

Anticorpi anti-ADAMTS13

Infezione gastrointestinale da ceppi di E. Coli produttori della tossina Shiga-like o S. Dysenteriae

Meccanismo immunomediato (es. Quinino) o effetto tossico dosedipendente (es. inibitori del VEGF)

Anticorpi anti-CFH 
TABELLA III - Fattori trigger della SEUa

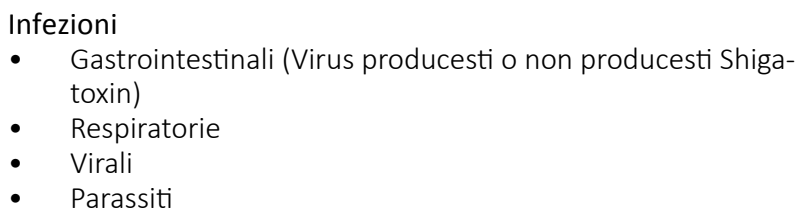

Farmaci e vaccinazioni

Gravidanza

Patologie autoimmuni

Neoplasie

Trapianto di midollo osseo

Radiazioni ionizzanti

CFI e THBD) e mutazioni tipo "gain of function" di C3 e CFB sono state identificate in pazienti affetti da SEUa. II gene più frequentemente coinvolto (più del $20-30 \%$ dei casi di SEUa) è quello che codifica per il Fattore $\mathrm{H}$ (CFH) sul cromosoma 1 (1q32); sono state descritte oltre 100 mutazioni (missense, nonsense, piccole delezioni/inserzioni), sia in omozigosi che in eterozigosi, determinanti un deficit quantitativo (tipo I) o qualitativo (tipo II) di CFH. Nel 4-10\% dei casi, invece, la causa è una mutazione del gene che codifica per il Fattore I (CFI) (4q25) e che determina un'alterata regolazione della cascata del complemento sia in fase solida che in fase liquida. Una prevalente iperattivazione complementare in fase solida è, invece, descritta in caso di mutazioni del gene che codifica per la Membrane Cofactor Protein (MCP) (1q32.2). Più rare sono, invece, le mutazioni della trombomodulina (THBD), del Fattore B (CFB) e del C3 i cui geni sono rispettivamente localizzati sui cromosomi 20 (20p11.21), 6 (6p21.33) e 19 (19p13.3). Inoltre, sono state descritte mutazioni che coinvolgono una sequenza di geni (CFHR3, CFHR1, CFHR4, CFHR2 e CFHR5) posti in prossimità del gene che codifica per CFH e che producono 5 proteine correlate con $\mathrm{CFH}$ : delezioni, in omozigosi o eterozigosi composta, di CFHR3/CFHR1 e CFHR1/CFHR4 sono state associate alla presenza di anticorpi anti-CFH, mentre riarrangiamenti tra CFHR1 e CFH oppure tra CFHR3 e CFH determinano la produzione di proteine ibride non funzionanti. Generalmente, le mutazioni possono interessare un singolo gene ma, nel $12 \%$ dei pazienti, è stata descritta la combinazione di due o più mutazioni (8). Le forme acquisite riconoscono un fattore scatenante (p. es., farmaci, malattie sistemiche, agenti virali o batterici diversi da STEC), tuttavia, anche in questo caso, sembra essere presente una predisposizione genetica. In assenza di mutazioni genetiche, riscontrate in circa il $60-70 \%$ di SEUa, o di una storia familiare suggestiva di SEUa o di fattori scatenanti (Tab. III), la patologia viene definita idio- patica. La SEUa è associata a un'elevata mortalità nella fase acuta e alla progressione, nel 50-60\% dei casi, a insufficienza renale terminale (ESRD) $(9,10)$.

La MAT metabolismo-mediata ha tipicamente esordio in età pediatrica ed è causata da un difetto ereditario del metabolismo della cobalamina (vitamina B12), che determina la formazione di agenti ossidanti, una disfunzione endoteliale, un'aumentata espressione di fattore tissutale e la conseguente attivazione della coagulazione. Tutte queste alterazioni sono responsabili dell'iperattivazione della via alterna della cascata del complemento, con la conseguente formazione di trombi nel microcircolo e danno d'organo. In questi casi, il trattamento si basa sulla somministrazione, per via parenterale, di idrossicobalamina.

Le forme di MAT associate a un'alterazione della coagulazione comprendono quella secondaria alla mutazione della diacilglicerolo chinasi $\mu$ (DGKE) e quella secondaria a mutazioni a carico di trombomodulina (THBD) e plasminogeno (PLG). La prima ha un esordio in età precoce, generalmente nel primo anno d'età; è provocata da una mutazione, in omozigosi o eterozigosi composta, del gene che codifica per DGKE (locus 17q22) (11) ed è trasmessa con modalità recessiva a penetranza completa. DGKE, in condizioni fisiologiche, svolge un ruolo anti-trombotico e la perdita della sua funzione nel corso di una MAT determina l'attivazione della proteina chinasi C (PKC). Ciò favorisce l'up-regulation di fattori pro-trombotici (VWF, TF e PAI 1) e la down-regulation del recettore del VEGF (Vascular Endothelial Growth Factor), che favoriscono uno stato pro-trombotico. In questi pazienti, il danno renale è preponderante ed è scarso il beneficio delle infusioni di plasma, associate o meno alla plasmaferesi, con una frequente evoluzione in insufficienza renale cronica. II meccanismo eziopatogenetico delle forme con deficit della THBD non è del tutto noto ma sembra implicare il coinvolgimento sia della cascata coagulativa sia di quella del complemento e, per tale motivo, è assimilabile alle forme complemento-mediate, suscettibili al trattamento con anticorpi anticomplementari.

Tra le forme acquisite di MAT, è descritta quella secondaria all'infezione da Escherichia Coli e Shigella dysenteriae (tipo 1). Il sierotipo 0157:H7 di E. Coli è quello che più frequentemente si associa a ST-SEU, tuttavia anche i sierotipi O111:H8, 0103:H2, O104:H4, 0121, 0145, 026 e 0113 possono esserne responsabili. I suddetti ceppi di $E$. Coli producono due possibili tipi di tossina (Stx1 e Stx2), molto simili alla potente tossina implicata nelle tossinfezioni da $S$. dysenteriae. I suddetti batteri colonizzano l'intestino dei bovini (raramente isolati anche in cervi, ovini, cavalli, cani e volatili), che, tuttavia, sono privi del recettore necessario per l'espletamento degli effetti della tossina. Quest'ultima viene trasmessa all'uomo in seguito all'assunzione di alimenti o acqua contaminata. II quadro microangiopatico si manifesta prevalentemente in età pediatrica (2-6 anni) dopo circa 24 ore dall'esordio della sintomatologia gastrointestinale (dolore addominale con diarrea acquosa e/o emorragica). La tossina è costituita da 
una subunità $A$ e da cinque subunità $B$; ciascuna delle subunità $B$ si lega con elevata affinità al recettore della globotriaosilceramide (Gb3 o CD77), espresso sulla superficie delle cellule endoteliali ma anche dalle cellule mesangiali, dai podociti e dalle cellule tubulari del rene, provocando il blocco della sintesi proteica con conseguente apoptosi e concomitante attivazione di uno stato pro-infiammatorio e pro-trombotico, oltre alla liberazione del vWF. La risoluzione del quadro clinico si osserva nel $75 \%$ dei pazienti (12) mentre la mortalità, in fase acuta, oscilla dal $3 \%$ al $5 \%$ (13).

Un'altra forma di MAT secondaria associata a un quadro infettivo è quella legata all'infezione da Streptococcus Pneumoniae; questo batterio produce una neuroaminidasi che rimuove i residui di acido sialico dalla membrana cellulare, esponendo l'antigene Thomsen-Friedenreich ad anticorpi preformati circolanti di classe IgM. Questo legame avviene sulla superficie piastrinica e sulle cellule endoteliali, determinando aggregazione piastrinica e danno endoteliale. II quadro microangiopatico, in questi casi, complica un'infezione pneumococcica e si manifesta generalmente in bambini di età inferiore ai 2 anni.

Un quadro di MAT acquisita può essere, inoltre, scatenato dall'assunzione di farmaci sia tramite un meccanismo immuno-mediato sia per il loro effetto tossico, dose-dipendente.

La formazione di anticorpi sembra essere responsabile della forma di MAT associata a chinino; essa si manifesta con un quadro di rinite, mialgia, vomito e oligoanuria, che insorge entro 12 ore dall'assunzione del farmaco, in pazienti precedentemente sensibilizzati; I'anemia è, in genere, di grado moderato. Altre forme immuno-mediate sembrano essere quelle da quetiapina e gemcitabina.

Una tossicità dose e tempo-mediata è stata, invece, descritta per i farmaci immunosoppressivi e gli inibitori del VEGF. In una review su 188 trapianti di rene, fu osservata un'incidenza di MAT indotta da ciclosporina (CyA) e tacrolimus (FK) rispettivamente nel $14 \%$ e nell' $1-5 \%$ dei casi (14). Gli inibitori della calcineurina sembrano agire tramite un danno diretto sull'endotelio, mediato dall'endotelina-1. Gli inibitori di mTOR, talvolta usati allo scopo di evitare l'effetto protrombotico degli inibitori della calcineurina, agiscono come trigger della SEUa tramite l'inibizione del VEGF.

L'incidenza di MAT in corso di terapia con mitomicina si aggira intorno al 2-10\%, avviene almeno un anno dopo l'inizio del trattamento e presenta un'elevata mortalità $(75 \%$ a 4 mesi) (15). II meccanismo patogenetico si basa su un danno diretto all'endotelio.

La presenza di anticorpi anti-ADAMTS13 è stata riscontrata in molti casi di MAT associati all'assunzione di agenti antipiastrinici (ticlopidina e clopidogrel); tuttavia, in alcuni casi, è stato descritto anche un possibile meccanismo di danno diretto sull'endotelio da parte di questi farmaci.

Forme di PTT, correlate con la presenza di anticorpi antiADAMTS13 in circolo, sono state osservate anche nel corso del trattamento con interferon-alfa (16) mentre non è ancora
TABELLA IV - Patologie che si possono manifestare con quadri di microangiopatia trombotica

Infezioni sistemiche

Neoplasie

Condizioni associate alla gravidanza (pre-eclampsia, eclampsia, HELLP syndrome)

Ipertensione maligna

Patologie autoimmuni (LES, SS, APS)

Trapianto di cellule staminali o d'organo

chiara la patogenesi nei casi di SEUa associati all'utilizzo del farmaco. In letteratura, sono riportati, inoltre, casi di SEUa associati a una terapia prolungata con interferon-beta, che sembra agire alterando la cascata del complemento oppure attraverso un danno diretto sull'endotelio; nessuna mutazione genetica è stata identificata in questi casi (17-21).

È stata descritta la correlazione della MAT con altri farmaci (cisplatino, bleomicina, OKT3, contraccettivi orali e FANS), tuttavia non sempre è chiaro il meccanismo patogenetico alla base.

La presenza di una delle suddette condizioni, di per sé, non è sufficiente per lo sviluppo della malattia e solo la concomitante presenza di fattori trigger (gravidanza, chirurgia o malattie infiammatorie) ne può scatenare l'esordio clinico. Inoltre esistono forme in cui il quadro di MAT è secondario a patologie sistemiche (Tab. IV) o ad altre condizioni fisiopatologiche. Malgrado il non sempre chiaro meccanismo patogenetico, questi casi spesso sono stati associati a mutazioni genetiche predisponenti (9) e, probabilmente, agiscono essi stessi come fattori trigger. Inoltre, il trattamento della patologia scatenante il quadro di MAT spesso determina il risolversi della sintomatologia (2).

Meccanismo patogenetico comune a tutte le forme descritte sembra essere l'iperattivazione della via alterna del sistema del complemento (Fig. 1) che avviene tramite meccanismi diversi: iperespressione della P-selectina sulle cellule endoteliali indotta dalla Shiga-toxin nella ST-SEU, mutazioni dei geni codificanti per molecole regolatrici della cascata del complemento o per componenti della C3-convertasi o la presenza di anticorpi anti-Fattore $\mathrm{H}$ nelle forme complemento-mediate e deficit dell'enzima ADAMTS13 nella PTT (22). L'attivazione del sistema del complemento, oltre a determinare la formazione del Complesso di Attacco alla Membrana (MAC o C5b9), stimola processi infiammatori (rilascio dei componenti del complemento contenuti nei depositi intracellulari da parte dei leucociti attivati e innesco della cascata indotta dai neutrofili $(23,24))$ ed emostatici (attivazione e aggregazione piastrinica e attivazione dei fattori della coagulazione $(25,26))$. In condizioni fisiologiche, l'innesco 


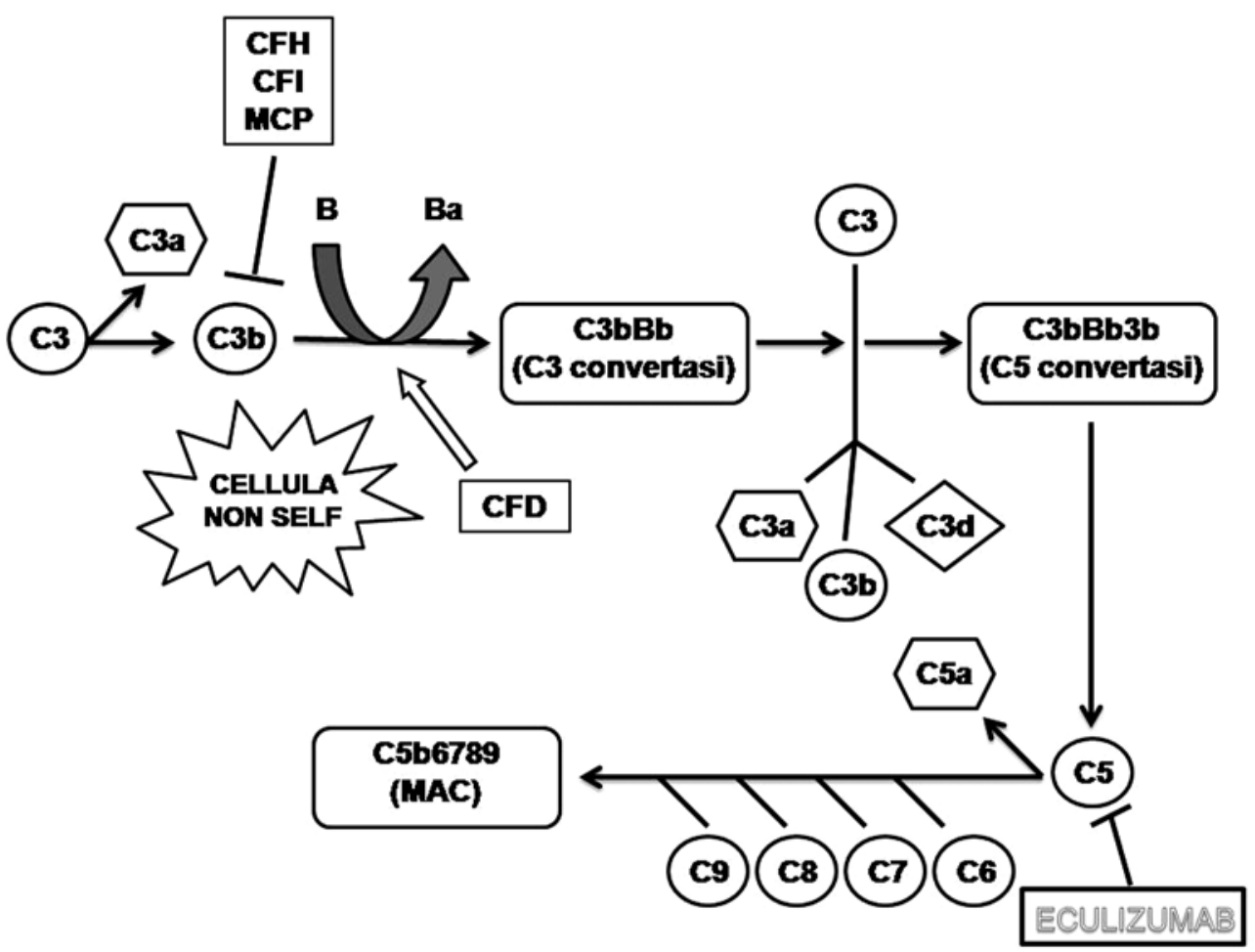

Fig. 1 - Via alterna del complemento. $\mathrm{CFH}$ : Fattore $\mathrm{H}$; CFI: Fattore I; MCP: Membrane Cofactor Protein; CFD: Fattore D; MAC: Membrane Attack Complex.

della cascata del complemento è controllato da una serie di molecole, ancorate alla superficie cellulare o circolanti, che prevengono la formazione del MAC, se non necessaria. L'attivazione non controllata del sistema del complemento, indipendentemente dalla causa, determina un persistente danno alle cellule endoteliali con esposizione della matrice subendoteliale, richiamo di leucociti e attivazione piastrinica con conseguente formazione di trombi. La trombocitopenia e l'anemia emolitica, caratteristiche di laboratorio in comune tra le forme descritte di MAT, sono determinate rispettivamente dall'aggregazione delle piastrine, che contribuiscono alla formazione dei microtrombi, e dalla frammentazione dei globuli rossi secondaria al loro passaggio attraverso il lume ristretto dei vasi del microcircolo. Dal punto di vista istologico, queste alterazioni si traducono nel distacco delle cellule endoteliali, che presentano un aspetto edematoso, dalla membrana basale, con accumulo subendoteliale di materiale elettronlucente, presenza di trombi piastrinici intraluminali e occlusione parziale o totale del lume vascolare; nelle fasi iniziali è, inoltre, possibile osservare aree di infiltrazione leucocitaria.

Le formazioni trombotiche che si riscontrano in corso di MAT si differenziano secondo il diverso meccanismo scatenante. Nella PTT i trombi sono costituiti prevalentemente da vWF e piastrine e localizzati nelle arteriole e nei capillari di numerosi organi. Le formazioni trombotiche in corso di SEU si caratterizzano, invece, per la presenza di fibrina, piastrine e globuli rossi, ma hanno una diversa localizzazione: a livello dei capillari glomerulari nella SEUa e delle arteriole renali e delle arterie interlobulari nella ST-SEU.

\section{Manifestazioni cliniche delle microangiopatie trombotiche}

L'esordio delle MAT è, generalmente, improvviso e associato a una sintomatologia aspecifica: febbre, sensazione di malessere generale e astenia. Talvolta, può essere preceduto da un quadro infettivo, più frequentemente a carico delle vie aeree superiori, ma, anche, gastrointestinale, o da infezioni virali (p. es., varicella, virus $\mathrm{N} 1 \mathrm{H} 1$ ), che svolgono un ruolo trigger della patologia. Non raramente il primo episodio di malattia si manifesta nel corso della gravidanza o nell'immediato post-partum.

Le principali manifestazioni clinico-laboratoristiche che si riscontrano in corso di MAT sono:

I. piastrinopenia: generalmente di grado severo $(<150 \times$ $10 \%$ L o riduzione di più del $25 \%$ rispetto ai valori iniziali) con presenza in circolo di piastrine giganti dovuta all'attivazione della trombocitopoiesi. L'emivita piastrinica è, invece, ridotta a causa della loro distruzione, che avviene a livello del microcircolo;

II. anemia emolitica microangiopatica con bassi valori di emoglobina $(\mathrm{Hb}<10 \mathrm{~g} / \mathrm{dL}$ ) che si associano a segni di emolisi: incremento di LDH, della bilirubina (prevalentemente la forma non coniugata) e dei reticolociti, con 
aptoglobina ridotta o indosabile, presenza di schistociti nel sangue periferico ( $>2 \%$ ) e test di Coombs, diretto e indiretto, negativo;

III. danno d'organo secondario alla formazione dei microtrombi. II coinvolgimento neurologico, frequentemente associato a una più probabile diagnosi di PTT, non è considerato sempre dirimente per la diagnosi di questa forma di MAT, come dimostrato dai dati del registro francese (27). Esso può essere riscontrato anche nel $10 \%$ dei pazienti affetti da SEUa (28) anche se, in questi casi, è prevalente il coinvolgimento renale. Quadri di interessamento renale, talvolta anche molto severi, possono essere osservati anche nelle forme acquisite di PTT. Per tale motivo, l'interessamento neurologico e quello renale, nonostante il primo sia più frequente nella PTT e il secondo nella SEUa, non possono essere utilizzati per distinguere le due forme di MAT. In letteratura, sono stati riportati, inoltre, casi con interessamento cardiaco (28), polmonare $(29,30)$, gastrointestinale $(9,31)$ o muscolare $(32,33)$. Infine, aneddotici, i casi di lesioni gangrenose delle dita di mani e piedi sono stati descritti nella popolazione pediatrica (34-36).

Frequentemente, alle suddette manifestazioni si associa un quadro di ipertensione arteriosa di grado severo, che può essere secondaria sia alla ritenzione di volume, nei pazienti oligo-anurici, sia all'iper-reninemia, conseguenza del danno renale indotto dalla microangiopatia. Valori elevati di pressione arteriosa possono essere responsabili di un quadro di scompenso cardiaco o di manifestazioni neurologiche (p. es., convulsioni).

Agli esami di laboratorio, oltre ai segni di emolisi e all'eventuale peggioramento degli indici di funzionalità renale associato o meno a proteinuria nei casi con coinvolgimento renale, è possibile riscontrare una riduzione dei valori di C3 con C4 nella norma, come segno di attivazione della via alterna del complemento.

\section{Diagnosi differenziale tre le varie forme di microangiopatia trombotica}

In considerazione della sovrapponibile presentazione clinica delle varie forme di MAT, dell'elevata mortalità a cui si associano (10-20\% per la PTT, $25 \%$ per la SEUa (37) e 0-5\% per la SEU tipica (38)) e, di conseguenza, della necessità di instaurare precocemente la terapia più adeguata, è necessario eseguire un'accurata diagnosi differenziale.

Un esordio in età pediatrica deve orientare la diagnosi verso forme di SEU tipica o forme familiari di PTT, SEUa o MAT metabolismo o complemento-mediate mentre un esordio in età più avanzata è più frequente per le forme acquisite di PTT o per le forme secondarie di SEUa.

Di fronte a un quadro laboratoristico suggestivo di MAT è opportuno eseguire un'approfondita storia clinica del paziente e dei suoi familiari, indagare la presenza di fattori scatenanti ed eseguire un accurato esame obiettivo. Inoltre, devono essere eseguiti approfondimenti per distinguere le principali forme.

L'isolamento della Stx-E. Coli nelle coprocolture o la positività della sierologia per Stx o per il lipopolisaccaride 0157 sono suggestivi di SEU tipica sia che il quadro sia preceduto da diarrea, frequentemente emorragica, sia nei casi con assenza di sintomatologia gastrointestinale (circa il $25 \%$ ).

La presenza di un attività di ADAMTS13 $\leq 5 \%$, soprattutto se associata a valori di creatininemia $\leq 2.26 \mathrm{mg} / \mathrm{dL}$ e a una conta piastrinica $\leq 30 \times 10^{9} / \mathrm{L}$, depone per una diagnosi di PTT. Il valore predittivo positivo per le forme acquisite di PTT sale dall' $85 \%$ al $98 \%$ in presenza di tutti e tre i suddetti parametri rispetto al deficit isolato dell'attività di ADAMTS13 (27). Da ricercare, inoltre, la presenza di anticorpi anti-ADAMTS13, più frequenti nei pazienti adulti e responsivi alla terapia immunosoppressiva.

La diagnosi di SEUa è più probabile nei casi in cui non sia stata dimostrata l'infezione da $E$. Coli e in cui i valori di ADAMTS13 risultino nella norma e il coinvolgimento renale sia preponderante.

La presenza di mutazioni genetiche, dimostrate nel 60-70\% dei casi di SEUa e nelle forme metabolismo e coagulazione-mediate di MAT, è, invece, utile solo per conferma diagnostica e a scopo prognostico, essendo disponibili tardivamente a causa dei lunghi tempi di esecuzione. Da indagare, infine, la presenza di anticorpi anti-CFH, responsabili del $6 \%$ dei casi di SEUa.

\section{Approccio al paziente adulto con microangiopatia trombotica}

In presenza di un quadro clinico-laboratoristico suggestivo di MAT, è opportuno instaurare un'adeguata terapia di supporto consensualmente agli opportuni approfondimenti diagnostici. Trasfusioni di emazie dovranno essere eseguite in caso di anemia, mentre è da evitare, se non in caso di effettiva necessità, la trasfusione di piastrine, poiché possibile aggravante del quadro di emolisi. Fondamentali, a scopo diagnostico, sono il campionamento di sangue prima dell'inizio della plasmaferesi per la determinazione dell'attività di ADAMTS13 e la ricerca dell'inibitore, oltre al dosaggio dei vari fattori implicati nella cascata del complemento.

In considerazione della gravità di queste patologie e della severa prognosi a cui sono correlate, è importante instaurare una terapia specifica il più precocemente possibile, anche per prevenire le eventuali complicanze e un danno d'organo permanente.

Recentemente, è stato proposto un algoritmo che, basandosi sulle caratteristiche cliniche e di laboratorio, oltre che sulla risposta al plasma-exchange, suggerisce un modello comportamentale nell'approccio a queste complesse patologie. In presenza di manifestazioni cliniche e di laboratorio suggestive per microangiopatia trombotica viene suggerito di 
iniziare la terapia con plasma-exchange. La risposta a questa terapia può essere parte integrante del percorso diagnostico: il miglioramento clinico in corso di plasma-exchange depone per una diagnosi di forma ereditaria di PTT. La mancata o incompleta risposta dopo 4-5 sedute in presenza di valori di attività enzimatica di ADAMTS13 inferiori al $5 \%$ suggerisce una probabile diagnosi di PTT acquisita e, quindi, la necessità di iniziare una terapia immunosoppressiva. Se, invece, l'attività enzimatica è nei limiti della normalità, l'approccio più corretto sembra essere l'inizio di una terapia con Eculizumab, anticorpo monoclonale diretto contro la frazione $\mathrm{C} 5$ del complemento, nel sospetto di SEUa (39).

\section{Conclusioni}

Le MAT sono un gruppo di patologie dal complesso approccio diagnostico e terapeutico. Fondamentale è il riconoscimento dei primi segni clinici e parametri di laboratorio, che frequentemente avviene nei centri di primo soccorso, allo scopo di indirizzare il paziente verso reparti specializzati per la diagnostica e il trattamento. Un corretto e precoce approccio permette un migliore outcome in termini di mortalità, oltre che una minore incidenza di complicanze. Inoltre, una maggiore conoscenza dei meccanismi patogenetici ha permesso, negli ultimi anni, l'introduzione di farmaci nuovi e con una maggiore specificità per la malattia, che garantiscono migliori risultati in caso di diagnosi corretta.

\section{Disclosures}

Financial support: No financial support was received for this submission.

Conflict of interest: The authors have no conflict of interest.

\section{Bibliografia}

1. Besbas N, Karpman D, Landau D, et al. A classification of hemolytic uremic syndrome and thrombotic thrombocytopenic purpura and related disorders. Kidney Int. 2006;70:423-31.

2. George JN, Nester CM, McIntosh JJ, et al. Syndromes of thrombotic microangiopathy. N Eng J Med. 2014;371:654-66.

3. Moschowitz E. Hyaline thrombosis of the terminal arterioles and capillaries: a hiterto undescribed disease. Proc N Y PAthol Soc. 1924;24:21-4.

4. Verweyen HM, Karch H, Brandis M, Zimmerhackl LB. Enterohemorrhagic Escherichia coli infections: following transmission routes. Pediatr Nephrol. 2000;14:73-83.

5. Caprioli J, Noris M, Brioschi S, et al. Genetics of HUS: the impact of MCP, CFH and IF mutations in clinical presentation, response to treatment, and outcome. Blood. 2006;108:1267-79.

6. Sellier-Leclerc AL, Fremeaux-Bacchi V, Dragon-Durey MA, et al. Differential impact of complement mutations on clinical characteristics in atypical hemolytic uremic syndrome. J Am Soc Nephrol. 2007;8:2392-400.

7. Sullivan M, Erlic Z, Hoffmann MM, et al. Epidemiological approach to identifying genetic predispositions for atypical hemolytic uremic syndrome. Ann Hum Genet. 2010;74:17-26.

8. Loirat C, Noris M, Fremeaux-Bacchi V. Complement and atypical hemolytic uremic syndrome in children. Pediatr Nephrol.
2008;23:1957-72.

9. Noris $M$, Caprioli J, Bresin E, et al. Relative role of genetic complement abnormalities in sporadic and familial a HUS and their impact on clinical phenotype. Clin J Am Soc Nephrol. 2010;5:1844-59.

10. Caprioli J, Noris M, Brioschi S, et al. Genetics of HUS: the impact of $\mathrm{MCP}, \mathrm{CFH}$ and IF mutations in clinical presentation, response to treatment, and outcome. Blood. 2006;108:1267-79.

11. Lemaire $M$, Frémeaux-Bacchi $V$, Schaefer $F$, et al. Recessive mutations in DGKE cause atypical hemolytic-uremic syndrome. Nat Genet 2013;45:531-6.

12. Noris M, Mescia F, Remuzzi G. STEC-HUS, atypical HUS and TTP are all disease of complement activation. Nat Rev Nephrol. 2012;8:622-633.

13. Garg AX, Suri RS, Barrowman N, et al. The long-term renal prognosis if diarrhea associated hemolytic uremic syndrome: $A$ systemic review, meta-analysis and meta-regression. J Am Soc Nephrol. 2003;14:299.

14. Noris M, Remuzzi G. Hemolytic uremic syndrome. J Am Soc Nephol. 2005;16:1035-50.

15. Zarifian A, Meleg-Smith S, O'donovan R, Tesi RJ, Batuman V. Cyclosporine-associated thrombotic microangiopathy in renal allografts. Kidney Int. 1999;55:2457-66.

16. Dlott JS, Danielson CF, Blue-Hnidy DE, McCarthy LJ. Drug-induced thrombotic thrombocytopenic purpura/hemolytic uremic syndrome: a coincise revew. Ther Apher Dial. 2004;8: 102-11.

17. Kitano K, Gibo Y, Kamijo A, et al. Thrombotic thrombocytopenic purpura associated with pegylated-interferon alpha-2a by ADAMTS 13 inhibitor in a patient with chronic hepatitis $\mathrm{C}$. Haematologica. 2006;91:ERC34.

18. Hansen $T$, New $D$, Reeve $R$, et al. Acute renal failure, systemic lupus erythematosus and thrombotic microangiopathy following treatment with beta-interferon for multiple sclerosis: case report and review of the literature. NDT Plus. 2009;2:466-8.

19. Broughton A, Cosyns JP, Jadoul M. Thrombotic microangiopathy induced by long-term interferon- $\beta$ theraphy for multiple sclerosis: a case report. Clin Nephrol. 2011;5:396-400.

20. Ubara $\mathrm{Y}, \mathrm{Hara} \mathrm{S}$, Takedatu $\mathrm{H}$, et al. Hemolytic uremic syndrome associated with beta-interferon therapy for chronic hepatitis $C$. Nephron. 1998;80:107-8.

21. Olea T, Díaz-Mancebo R, Picazo ML, Martínez-Ara J, Robles A, Selgas $R$. Thrombotic microangiopathy associated with use of interferon-beta. Int J Nephol Renovasc Dis. 2012;5:97-100.

22. Hunt D, Kavanagh D, Drummond I, et al. Thrombotic microangiopathy associated with interferon-beta. N Eng J Med. 2014; 370:1270-1.

23. Noris M, Mescia F, Remuzzi G. STEC-HUS, atypical HUS and TTP are all disease of complement activation. Nat Rev Nephrol. 2012;8:622-33.

24. Høgåsen AK, Würzner R, Abrahamsen TG, Dierich MP. Human polymorphonuclear leukocytes store large amounts of terminal complement components $\mathrm{C} 7$ e $\mathrm{C} 6$, wich may be released on stimulation. J Immunol. 1995;154:4724-40.

25. Botto $M$, Lissandrini $D$, Sorio $C$, Walport MJ. Byosintesis and secretion of complement component (C3) by activated human polymorphonuclear leukocytes. J Immunol. 1992;149:1348-55.

26. Vogt W. Complement activation by myeloperoxidase products released from stimulated human polymorphonuclear leukocytes. Immunobiology. 1996;195:334-46.

27. Camous L, Roumenina L, Bigot S, et al. Complement alternative pathway acts as a positive feedback amplification of neutrophil activation. Blood. 2011;117:1340-9.

28. Coppo P, Schwarzinger M, Buffet $M$, et al. Predictive features 
of severe adquired ADAMTS13 deficiency in idiopathic thrombotic microangiopathies: the French TMA reference centre experience. PLoS ONE. 2010;5(4):e10208.

29. Neuhaus TJ, Calonder S, Leumann EP. Heterogeneity of atypical haemolytic uraemic syndromes. Arch Dis Child. 1997;76: 518-21.

30. Losito A, Pittavini L, Covarelli C. Thrombotic microangiophatic nephropathy, pulmonary hypertension and nephromegaly: case report of a patient treated with endothelin receptor antagonist. Clin Nephrol. 2012;77:164-70.

31. Abdallah E, Al-Helal B, Al-Rashidi A, Asad R. ANCA negative pulmonary renal syndrome with pathologic findings of thrombotic microangiopathy. Arab J Nephrol Transplant. 2013;6:31-5.

32. Sellier-Leclerc AL, Fremeaux-Bacchi V, Dragon-Durey MA, et al. Differential impact of complement mutations on clinical characteristics in atipical hemolytic uremic syndrome. J Am Soc Nephol. 2007;18:2392-400.

33. Pena DR, Vaccarello M, Neiberger RE. Severe hemolytic uremic syndrome associated with rhabdomyolysis and insulin-dependent diabetes mellitus. Child Nephrol Urol. 1991;11:223-7.

34. Andreoli SP, Bergstein JM. Acute rhabdomyolysis associated with hemolytic-uremic syndrome. J Pediatr. 1983;103:78-80.
35. Kaplan BS, Garcia CD, Chesney RW, Segar WE, Giugno K, Chem R. Peripheral gangrene complicating idiopathic and recessive hemolytic uremic syndromes. Pediatr Nephrol. 2000;14: 985-9.

36. Malina M, Gulati A, Bagga A, Majid MA, Simkova E, Schaefer F. Peripheral gangrene in children with atypical hemolytic uremic syndrome. Pediatrics. 2013;131:331-5.

37. Ozel A, Calişkan U, Gücer S. Peripheral gangrene complicating hemolytic uremic syndrome in a child. Pediatr Nephrol. 2003;18:465-7.

38. Scully M, Goodship T. How I treat thrombotic thrombocytopenic purpura and atypical haemolytic uraemic syndrome. $\mathrm{Br} J$ Haematol. 2014;164:759-66.

39. Verweyen HM, Karch H, Brandis M, Zimmerhackl LB. Enterohemorrhagic Escherichia coli infections: following transmission routes. Pediatr Nephrol. 2000;14:73-83.

40. Cataland SR, Wu HM. Diagnosis and management of complement mediated thrombotic microangiopathies. Blood Rev. 2014; 28(2):67-74.

41. Neuhaus TJ, Calonder S, Leumann EP. Heterogeneity of atypical haemolytic uraemic syndromes. Arch Dis Child. 1997;76:518-21. 